\title{
Transverse vibration of stiffened plates with cutouts subjected to in-plane uniform edge loading at the plate boundary
}

\author{
A.K.L. Srivastava ${ }^{\mathrm{a}, *}$, P.K. Datta ${ }^{\mathrm{b}}$ and A.H. Sheikh ${ }^{\mathrm{c}}$ \\ ${ }^{a}$ Applied Mechanics Department, N.I.T. Jamshedpur-831014, India \\ ${ }^{\mathrm{b}}$ Aerospace Engineering Department, I.I.T. Kharagpur-721302, India \\ ${ }^{\mathrm{c}}$ Department of Ocean Engineering, I.I.T. Kharagpur-721302, India
}

Received 3 June 2002

Revised 26 September 2002

\begin{abstract}
Vibration characteristics of stiffened plates with cutouts subjected to uni-axial in-plane uniform edge loading at the plate boundaries are investigated using the finite element method. The characteristic equations for the natural frequencies, buckling loads and their corresponding mode shapes are obtained from the equation of motion. The vibration frequencies and buckling load parameters for various modes of stiffened plates with cutouts have been determined for simply supported and clamped edge boundary conditions. In the structure modelling, the plate and the stiffeners are treated as separate elements where the compatibility between these two types of elements is maintained. Numerical results are presented for a range of hole to plate width ratios of 0 to 0.8 . The correlations of the natural frequencies and buckling parameters obtained by the present approach with those available in the literature are found to show good agreement.
\end{abstract}

Keywords: Finite element method, buckling, vibration, cutout

\section{Introduction}

Cutouts in aerospace, civil, mechanical and marine structures are inevitable mainly for practical considerations. In aerospace structures, cutouts are commonly found as access ports for mechanical and electrical systems, or simply to reduce weight. Cutouts in wing spar and cover panels of commercial transport and military aircrafts are needed to provide access for hydraulic lines and for damage inspection. In addition, the designers often need to incorporate cutouts or openings in a structure to serve as doors and windows. The buckling and vibration analysis of structures with cutouts pose a tremendous challenge and must be properly understood in the structural design. The instability effects are improved with the provision of stiffeners.

${ }^{*}$ Corresponding author: E-mail: rsaksriv@ aero.iitkgp.ernet.in.
Analyses of stiffened plates are carried out normally by energy methods by adding energies due to the plate and the stiffeners. An eight noded isoparametric stiffened plate-bending element for the free vibration analysis of stiffened plate has been presented by Mukherjee and Mukhopadhyay [9]. Here the stiffener can be positioned anywhere within the plate element and need not necessarily be placed on the nodal lines.

Olson and Hazell [11] have presented a critical study on clamped integrally stiffened plate by the finite element method. The mode shapes and frequencies have been determined experimentally using the real time holographic technique. The effect of change in rib stiffness on various modes has been studied. Buckling and vibration characteristics of stiffened plate subjected to in-plane partial edge loading has been studied by Srivastava et al. [19]. In the formulation, the stiffener can 
be positioned anywhere within the plate element and follow the plate beam idealization approach.

The numerical method for computing the natural frequencies of rectangular plates with cutouts can be broadly classified into three categories, namely finite element and finite difference methods, a series type analytical method and the semi analytical approach based on the Rayleigh-Ritz.

Numerical results obtained by the finite element method have been reported by Ali and Atwal [1], Shastry and Rao [16], Reddy [14] and Laura et al. [5]. Ali and Atwal [1] studied the natural frequencies of simply supported rectangular plates and rectangular cutouts using the Rayleigh-Ritz method. Paramsivam [12] used a finite difference approach in analyzing the effects of openings on the fundamental frequencies of plates with simply supported and clamped boundary conditions. A finite element analysis of clamped thin plates with different cutout sizes, along with experiments was carried out by Monahan et al. [8]. Mundkur et al. [10] studied the vibration of square plates with square cutouts by using boundary characteristics orthogonal polynomials satisfying the boundary conditions. Chang and Chiang [2] studied the vibration of the rectangular plate with an interior cutout by using the finite element method. Lee et al. [6] predicted the natural frequencies of rectangular plates with an arbitrarily located rectangular cutout. Ritchie and Rhodes [15] have investigated theoretically and experimentally the behaviour of simply supported uniformly compressed rectangular plates with central holes, using a combination of Rayleigh-Ritz and finite element methods.

Studies on vibration characteristics of stiffened plates with cutouts are scanty in the literature. The free vibration characteristics of unstiffened and longitudinally stiffened square panels with symmetrically square cutouts are investigated by Sivasubramonian et al. [17] using the finite element method. A rectangular shell element with seven degrees of freedom per node together with a beam element of seven degrees of freedom per node is used for the analysis. Studies are carried out on unstiffened as well as stiffened panels keeping the size, weight and boundary conditions the same. Lam and Hung [4] studied the vibrations of plates with stiffened openings using orthogonal polynomials and partitioning method. The method involves partitioning of the plate domain into appropriate rectangular segments in order to approximate the deflection function of each segment by sets of orthogonal polynomials. Natural frequencies of simply supported and fully clamped plates with stiffened openings are presented. The ef- fects of flexural rigidity and corresponding mass inertia of the stiffener upon the fundamental frequency of the structure are investigated.

Paramsivam and Sridhar Rao [13] modified the grid framework model suitably to obtain the natural frequencies of a square plate with stiffened square openings. This modelling has an advantage of using variation of stiffener geometry and intermediate beam support on the plate. This investigation provides a general feeling about the changes in fundamental frequencies that occur when stiffeners are introduced.

Recently, dynamic instability of stiffened plates subjected to harmonic in-plane uniform edge loading has been studied by Srivastava et al. [18] considering and neglecting in-plane displacements. The element matrices of the stiffened plate element consist of the contribution of the plate and that of the stiffener. The contribution of the beam element is reflected in all nodes of the plate element, which contains the stiffener. Further Srivastava et al. [20] extended their work to study the principal dynamic instability behaviour of stiffened plates subjected to non-uniform harmonic in-plane edge loading. The plate skin and the stiffeners are modelled as separate elements but the compatibility between them is maintained.

The present paper deals with the effects of various parameters such as size and location of cutouts, aspect ratios of the plate and cutout, different boundary conditions and stiffener parameters on buckling and vibration characteristics of rectangular stiffened plates with cutouts. The finite element is applied to analyze the vibration and the buckling behaviour of stiffened plates with cutouts subjected to in-plane uniform edge loading at the plate boundary. A nine-nodded isoparametric quadratic element with its ability to accommodate curved boundaries is selected for the modelling of the stiffened plates element with cutouts. Furthermore as the element considers shear deformation, the formulation is applicable to both thin and thick plates. The main elegance of the formulation lies in the treatment of stiffeners. In order to improve upon the earlier work, a more economic element is chosen in which the stiffener can be placed anywhere within the plate element which, helps to increase considerable amount of flexibility in the mesh generation. The formulation has been generalized to cater rectangular stiffened plates with rectangular cutouts having eccentric stiffener longitudinally or transversely. 


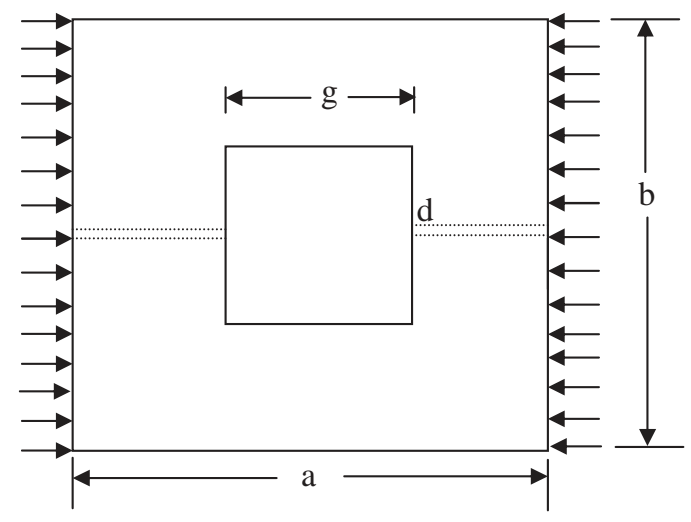

Fig. 1. Stiffened plates with cutout under in plane uniform edge loading at plate boundary.

\section{Description of the problem}

The problem considered here consists of a rectangular plate $(\mathrm{a} \times \mathrm{b})$ with stiffeners having a rectangular cutout of size $(g \times d)$ at the center as shown in Fig. 1. The plate with stiffener subjected to in-plane uniform edge loading at the plate boundary and stiffener cross-section are shown in Fig. 2. The loading applied is compressive in nature. All the boundaries of the plates are simply supported unless otherwise stated. The length (a) of the stiffened plate considered above is varied keeping its other parameters unchanged.

\section{Finite element formulation}

The formulation is based on Mindlin's plate theory, which will allow for the incorporation of shear deformation. The plate skin and the stiffeners are modelled as separate elements but the compatibility between them is maintained. The element matrices of the stiffened plate element consist of the contribution of the plate and that of the stiffeners. The effect of in-plane deformations is taken into account in addition to the deformations due to bending. This is similar to the concept proposed by Mukherjee and Mukhopadhyay [9]. A nine-noded isoparametric quadratic element with five degrees of freedom $\left(u, v, w, \theta_{x}\right.$, and $\left.\theta_{y}\right)$ per node is employed in the present analysis.

The elastic stiffness matrix $\left[K_{p}\right]$, geometric stiffness matrix $\left[K_{G p}\right]$ and mass matrix $\left[M_{p}\right]$ of the plate element may be expressed as follows.

$$
\left[K_{p}\right]=\int_{-1}^{+1} \int_{-1}^{+1}\left[B_{p}\right]^{T}\left[D_{p}\right]\left[B_{p}\right]\left|J_{p}\right| d \xi d \eta
$$

$$
\begin{aligned}
& {\left[K_{G p}\right]=} \\
& \int_{-1}^{+1} \int_{-1}^{+1}\left[B_{G p}\right]^{T}\left[\sigma_{p}\right]\left[B_{G p}\right]\left|J_{p}\right| d \xi d \eta \\
& {\left[M_{p}\right]=\int_{-1}^{+1} \int_{-1}^{+1}[N]^{T}\left[m_{p}\right][N]\left|J_{p}\right| d \xi d \eta}
\end{aligned}
$$

where

$$
\begin{aligned}
& {\left[B_{P}\right]=\left[\left[\begin{array}{lllll}
\left.B_{P}\right]_{1}\left[B_{P}\right]_{2} & \ldots & {\left[B_{P}\right]_{r}} & \ldots & {\left[B_{P}\right]_{9}}
\end{array}\right]\right.} \\
& {\left[B_{G P}\right]=\left[\left[B_{G P}\right]_{1}\left[B_{G P}\right]_{2} \ldots\left[B_{G P}\right]_{r} \ldots\right.} \\
& \left.\left[B_{G P}\right]_{9}\right]
\end{aligned}
$$

The elastic stiffness matrix $\left[K_{S}\right]$, geometric stiffness matrix $\left[K_{G S}\right]$ and mass matrix $\left[M_{S}\right]$ of a stiffener element placed anywhere within a plate element and oriented in the direction of $x$ may be expressed, in a manner similar to those of the plate element as follows:

$$
\begin{aligned}
& {\left[K_{S}\right]=\int_{-1}^{+1}\left[B_{S}\right]^{T}\left[D_{S}\right]\left[B_{S}\right]\left|J_{S}\right| d \xi} \\
& {\left[K_{G S}\right]=\int_{-1}^{+1}\left[B_{G S}\right]^{T}\left[\sigma_{S}\right]\left[B_{G S}\right]\left|J_{S}\right| d \xi} \\
& {\left[M_{S}\right]=\int_{-1}^{+1}[N]^{T}\left[m_{S}\right][N]\left|J_{S}\right| d \xi}
\end{aligned}
$$

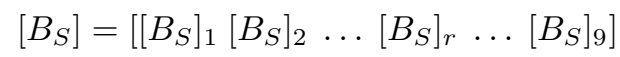

$$
\begin{aligned}
& {\left[B_{G S}\right]=\left[\left[B_{G S}\right]_{1}\left[B_{G S}\right]_{2} \ldots\left[B_{G S}\right]_{r} \ldots\right.} \\
& \left.\left[B_{G S}\right]_{9}\right]
\end{aligned}
$$

The different matrices in the above equations may be written as follows:

$$
\begin{gathered}
{\left[B_{S}\right]_{r}=\left[\begin{array}{ccccc}
\frac{\partial N_{r}}{\partial x} & 0 & 0 & 0 & 0 \\
0 & 0 & 0 & -\frac{\partial N_{r}}{\partial x} & 0 \\
0 & 0 & 0 & 0 & \frac{\partial N_{r}}{\partial x} \\
0 & 0 & \frac{\partial N_{r}}{\partial x} & -N_{r} & 0
\end{array}\right]} \\
{\left[D_{S}\right]=\left[\begin{array}{cccc}
E A_{S} & E F_{S} & 0 & 0 \\
E F_{S} & E I_{S} & 0 & 0 \\
0 & 0 & G T_{S} & 0 \\
0 & 0 & 0 & G A_{S} / 1.2
\end{array}\right]}
\end{gathered}
$$

The equation of equilibrium for the stiffened plate subjected to in-plane loads can be written as:

$$
[M]\{\ddot{q}\}+\left[\left[K_{b}\right]-P\left[K_{G}\right]\right]\{q\}=0
$$




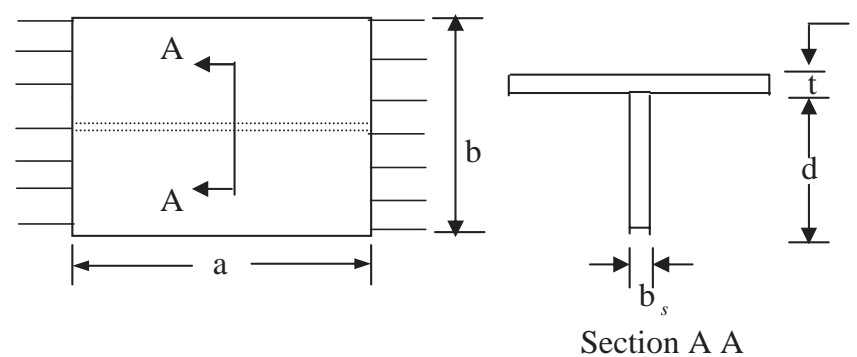

Fig. 2. Stiffened plate cross-section.

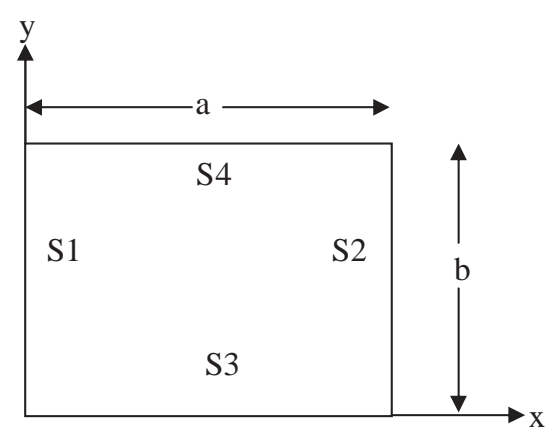

Fig. 3. Details of edges.

Equation (13) can be reduced to the governing equations for buckling and vibration problems.

A computer program is developed to perform all the necessary computations. The element matrices of the stiffened plate element consist of the contribution of the plate and that of the stiffener. A study of the stiffness and mass matrices of the stiffener element reveals that the contribution of the beam element is reflected in all 9 nodes of the plate element, which contains the stiffener. This is achieved through the expression of the strain displacement relationship in terms of displacement of 9 nodes of the plate. The contribution of the stiffener to a particular node depends on the proximity of the stiffener to that node. The whole domain of interest (plate area) is divided into a number of finite elements. For a given edge loading and boundary conditions, the static equation, i.e. $[K]=\{\delta\}=\{F\}$ is solved to get the stresses. The geometric stiffness matrix is now constructed with the known stresses. The overall elastic stiffness matrix, geometric stiffness matrix and mass matrix are generated from the assembly of those element matrices and stored in a single array where the variable bandwidth profile storage scheme is used. The solution of eigenvalues is performed by the simultaneous iteration technique proposed by Corr and Jennings [3].

\section{Numerical result and discussion}

Numerical results are presented for isotropic stiffened plates with cutouts for simply supported and clamped boundary conditions. In the discussion that followed, S, C denote simply supported, clamped respectively. The notation SCSC identifies a plate with the edges: $x=0, x=a, y=0, y=b$ having the boundary conditions in that order (Fig. 3). In Fig. 3, $\mathrm{S} 1, \mathrm{~S} 2, \mathrm{~S} 3, \mathrm{~S} 4$ are represented for side 1, side 2, side 3 and side 4 respectively. For comparison problems, the boundary conditions are considered as reported in the respective studies.

Majority of the model parameters and results are presented in non-dimensional form to make them independent of the plate size, thickness, cutout size, material properties, etc. The non-dimensionalisation of different parameters like vibration, buckling for stability analysis is taken as given below.

Frequency parameter $\frac{(\omega)=}{\omega b^{2} \sqrt{\rho t / D}}$ and Buckling parameter $(\lambda)=N_{X} b^{2} / \pi^{2} D$, where $D$ is the plate flexural rigidity, $D=E t^{3} / 12\left(1-\nu^{2}\right), \rho$ is the density of the plate material and $t$ is the plate thickness. Assuming a general case of several longitudinal ribs and denoting by $E I_{S}$ the flexural rigidity of a stiffener at a distance $\left(D_{x}\right)$ from the edge $y=0$, the stiffener parameter terms $\delta$ and $\gamma$ are defined as: $\delta=A_{S} / b t=$ Ratio of crosssectional area of the stiffener to the plate, where $A_{S}$ is the area of the stiffener. $\gamma=E I_{S} / b D=$ Ratio of bending stiffness rigidity of stiffener to the plate, where $I_{S}$ is the moment of inertia of the stiffener cross-section about reference axis. $g / a=$ Ratio of cutout to plate width.

\subsection{Convergence studies}

In finite element analysis, it is desirable to have the convergence studies to estimate the order of mesh size to be necessary for numerical solution. For this pur- 
Table 1

Convergence of frequency parameter for isotropic square plate

\begin{tabular}{lcclllll}
\hline $\begin{array}{l}\text { Boundary } \\
\text { condition }\end{array}$ & $\begin{array}{c}\text { Vibration } \\
\text { mode }\end{array}$ & $4 \times 4$ & $6 \times 6$ & $8 \times 8$ & $10 \times 10$ & $12 \times 12$ & $\begin{array}{r}\text { Leissa } \\
{[1989]}\end{array}$ \\
\hline SSSS & 1 & 19.757 & 19.741 & 19.738 & 19.737 & 19.737 & 19.74 \\
& 2 & 49.941 & 49.464 & 49.379 & 49.346 & 49.346 & 49.35 \\
& 3 & 49.941 & 49.464 & 49.378 & 49.346 & 49.346 & 49.35 \\
CCCC & 1 & 36.111 & 36.00 & 35.990 & 35.983 & 35.983 & 35.99 \\
& 2 & 75.330 & 73.789 & 73.511 & 73.404 & 73.404 & 73.41 \\
& 3 & 75.330 & 73.789 & 73.512 & 73.404 & 73.404 & 73.41 \\
\hline
\end{tabular}

Table 2

Natural frequency parameter of simply supported plate

\begin{tabular}{|c|c|c|c|c|c|c|}
\hline \multirow{3}{*}{ Mode } & \multicolumn{6}{|c|}{ Natural frequency parameter $(\omega)$} \\
\hline & \multicolumn{6}{|c|}{ Cut out ratio $(g / a)$} \\
\hline & 0 & 0.1 & 0.2 & 0.3 & 0.5 & 0.8 \\
\hline \multirow[t]{2}{*}{1} & 19.735 & 19.86 & 20.19 & 20.87 & 23.45 & 58.91 \\
\hline & $(19.7392)$ & (19.87) & $(20.193)$ & $(20.6983)$ & $(24.243)$ & $(58.358)$ \\
\hline \multirow[t]{2}{*}{2} & 49.22 & 49.35 & 49.492 & 49.514 & 48.40 & 73.83 \\
\hline & $(49.348)$ & $(49.355)$ & $(49.416)$ & $(49.512)$ & (48.409) & $(76.012)$ \\
\hline \multirow[t]{2}{*}{3} & 49.22 & 49.35 & 49.492 & 49.514 & 48.40 & 73.83 \\
\hline & $(49.348)$ & $(49.355)$ & $(49.416)$ & $(49.512)$ & $(48.409)$ & $(76.012)$ \\
\hline \multirow[t]{2}{*}{4} & 78.83 & 78.145 & 78.04 & 77.12 & 73.67 & 121.92 \\
\hline & $(78.956)$ & $(78.427)$ & (77.152) & $(76.052)$ & (76.58) & (126.71) \\
\hline
\end{tabular}

Parenthesis is for Mundkur et al. [10].

Table 3

Comparison of natural frequency parameter

\begin{tabular}{lccccc}
\hline \multirow{2}{*}{$g / a$} & \multicolumn{2}{c}{$\begin{array}{c}\text { Natural frequency parameter }(\omega) \\
\text { SSSS }\end{array}$} & & \multicolumn{2}{c}{ CCCC } \\
\cline { 2 - 3 } \cline { 5 - 6 } & Mundkur et al. [10] & Present & & Mundkur et al. [10] & Present \\
\hline 0.0 & 19.739 & 19.732 & & 35.985 & 35.981 \\
0.167 & 20.070 & 19.87 & & 37.425 & 36.06 \\
0.33 & 20.9633 & 20.12 & & 43.867 & 43.02 \\
0.5 & 24.2434 & 24.24 & & 65.715 & 65.27 \\
\hline
\end{tabular}

pose a simply supported and a clamped square plate subjected to in-plane uniform edge loading $(\mathrm{Nx})$ are analyzed with various mesh sizes. The results obtained are presented in Table 1 with those of Leissa and Ayoub [7]. It is observed that the frequency parameter results converged and agreed well with the above reference. As the convergence study shows that a mesh size of $10 \times 10$ is sufficient enough to get a reasonable order of accuracy, the analysis in the subsequent problems is carried out with this mesh size.

\subsection{Vibration studies of unstiffened plates}

In order to validate the results, linear fundamental frequency parameter of a simply supported isotropic square plate with various sizes of rectangular cutout (g/a) are computed and compared in Table 2 and for simply supported and clamped edges in Table 3 with Mundkur et al. [10] given in bracket, showing good agreement. Mundkur et al. [10] analyzed assuming boundary characteristics orthogonal polynomial functions, as assumed plate deflection shape functions satisfying the boundary conditions along the plate outer edges. The orthogonal polynomials are generated along both $\mathrm{x}$ and y directions.

The fundamental frequency parameter for square plate with square opening having different boundary conditions (SSSS, CCCC, CSCS) are studied in Figs 46.

The trend of the results for a simply supported plate shown in Fig. 4 are almost similar to the tends in Fig. 5 shown for CCCC plates, except for its gradient of rise when the cut-out size increases. For CCCC plate, there is a steep increase in frequencies with increasing cutout size. The increase in the natural frequency is more dominant in the CCCC case. When the plate cutout size $g / a$ is 0.8 , the fundamental frequency parameter is ten times that of the value with no cutout. However, the 


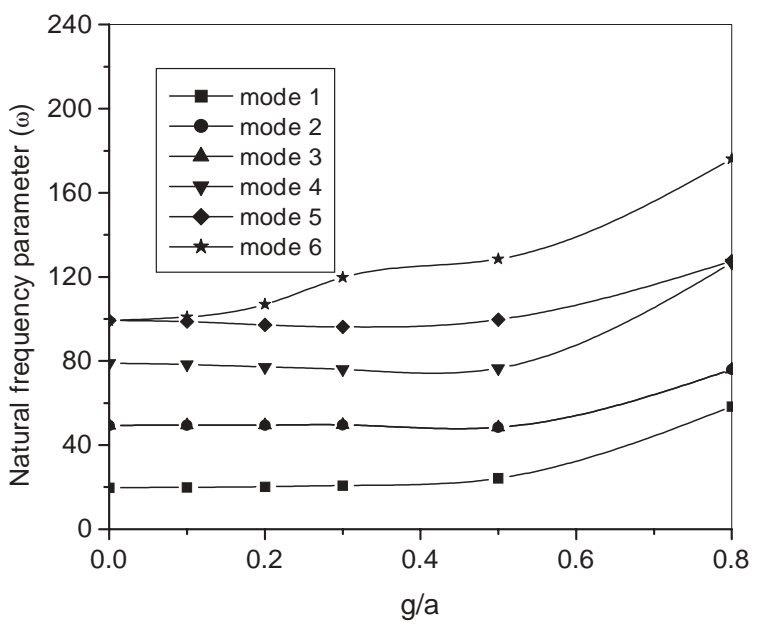

Fig. 4. The natural frequency parameter of a simply supported plate.

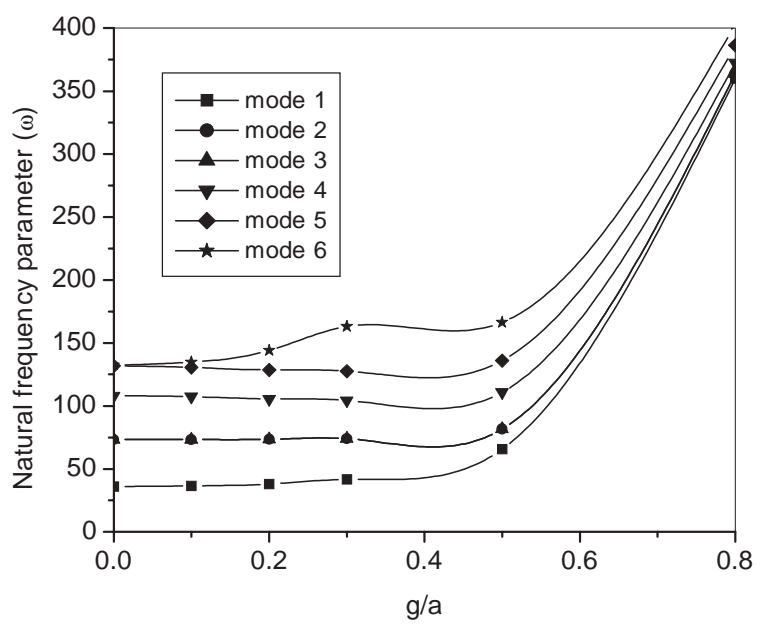

Fig. 5. As Fig. 4, but for clamped edges.

increase in higher natural frequencies is smaller. The fundamental frequency parameters for a square plate subjected to uniformly distributed biaxial compressive forces are studied and presented in Fig. 7. The natural frequencies are found to increase with decreased magnitude of compressive in-plane forces.

\subsection{Vibration studies of stiffened plates}

\subsubsection{Convergence and Validation studies}

An all side clamped and centrally stiffened square plate having a thickness of $1.37 \mathrm{~mm}$ is analyzed and compared with Mukherjee and Mukhopadhyay [9] and Olson and Hazell [11]. A stiffener of $6.35 \mathrm{~mm}$ in width and $12.7 \mathrm{~mm}$ in height is centrally placed in the plate. Results given in the Table 4 agree well with the theoret-

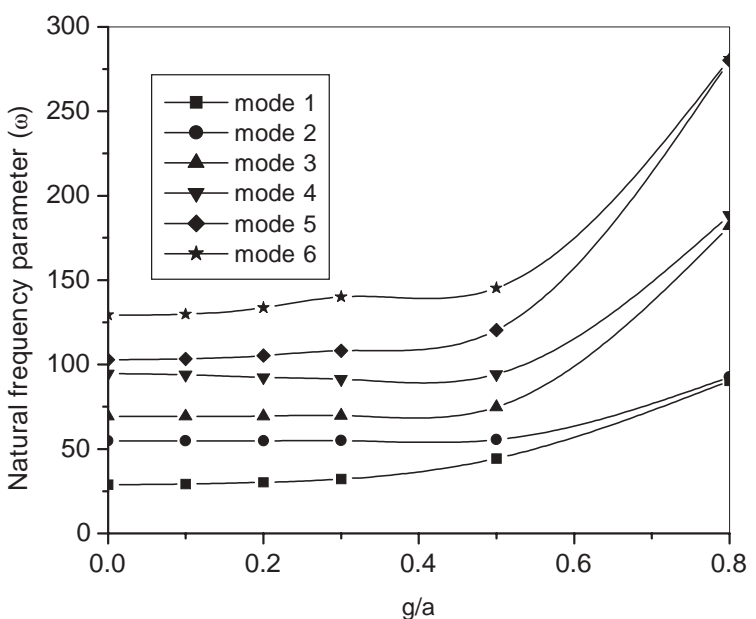

Fig. 6. As Fig. 4, but for CSCS boundary condition.

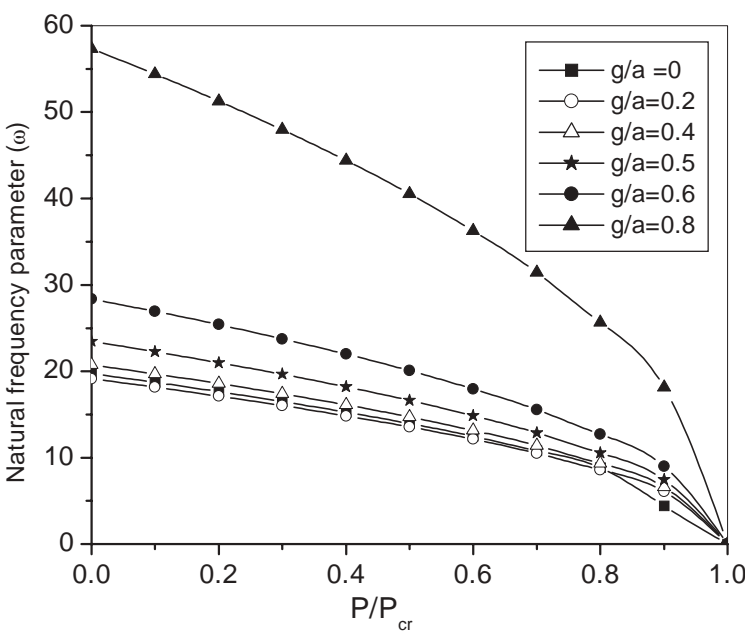

Fig. 7. Fundamental frequency parameter for a square plate with a square cutout subjected to bi-axial compressive forces.

ical values of Olson and Hazell [11]. Computation carried out by Mukherjee and Mukhopadhyay [9] is on the higher side with Olson and Hazell [11] except for the fundamental mode. However this theory indicates that the percentage difference between the present formulation and other two theories are within 5\%. Mukherjee and Mukhopadhyay [9] have used eight noded isoparametric plate-bending elements for finite element analysis. Here the stiffener can be positioned anywhere within the plate element and need not necessarily be placed on the nodal lines.

Olson and Hazell [11] have presented a critical study on clamped integrally stiffened plate by the finite element method. The mode shapes and frequencies have been determined experimentally using the real time 
Table 4

Frequencies for all sided clamped plate with central stiffener. Size of the plate $=203 \mathrm{~mm} \times 203 \mathrm{~mm} \times 1.37 \mathrm{~mm}$ thickness, Size of the stiffener $=6.35 \mathrm{~mm} \times 12.7 \mathrm{~mm} . E=7020 \mathrm{Kg} / \mathrm{mm}^{2}, \nu=0.3$

\begin{tabular}{ccccr}
\hline Mode & \multicolumn{2}{c}{ Olson and Hazel [11] } & Mukhopadhyay & Present \\
\cline { 2 - 3 } & Theory & Experiment & & \\
\hline 1 & 718.1 & 689.0 & 711.8 & 715.2 \\
2 & 751.4 & 725.0 & 768.2 & 741.9 \\
3 & 997.4 & 961.0 & 1016.5 & 1011.5 \\
4 & 1007.1 & 986.0 & 1031.9 & 1025.7 \\
5 & 1419.8 & 1376.0 & 1465.2 & 1451.8 \\
\hline
\end{tabular}

Table 5

Convergence and validation of non-dimensional fundamental frequencies of a SSSS and CCCC unstiffened / stiffened square plates with cutout. $g / a=0.5, a / b=1, b / h=100, \gamma=10$

\begin{tabular}{|c|c|c|c|c|c|c|c|c|}
\hline \multirow[t]{3}{*}{ Boundary condition } & \multirow[t]{3}{*}{ Types of plate } & \multicolumn{7}{|c|}{ Non-dimensional fundamental frequencies } \\
\hline & & \multicolumn{5}{|c|}{ Mesh size } & \multirow{2}{*}{$\begin{array}{c}\text { Ref } \\
{[1977]}\end{array}$} & \multirow{2}{*}{$\begin{array}{c}\text { Ref } \\
{[1973]}\end{array}$} \\
\hline & & $4 \times 4$ & $6 \times 6$ & $6 \times 8$ & $10 \times 10$ & $12 \times 12$ & & \\
\hline \multirow[t]{2}{*}{ Simply supported } & Unstiffened & 26.89 & 26.87 & 26.57 & 26.42 & 26.48 & 26.80 & 25.45 \\
\hline & Stiffened & 30.98 & 30.79 & 30.12 & 30.02 & 30.02 & 29.02 & 28.72 \\
\hline \multirow[t]{2}{*}{ Clamped } & Unstiffened & 67.09 & 66.92 & 66.80 & 66.80 & 66.81 & 68.67 & 57.25 \\
\hline & Stiffened & 71.12 & 71.09 & 70.98 & 70.57 & 70.56 & 70.87 & 68.62 \\
\hline
\end{tabular}

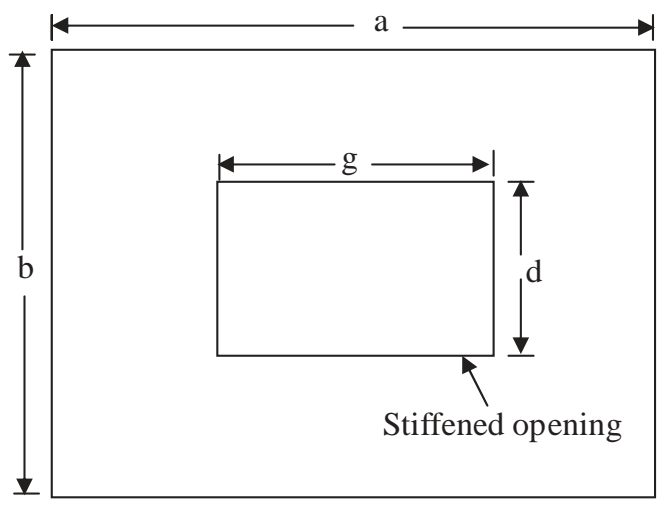

Fig. 8. Rectangular plate with rectangular stiffened opening.

holographic technique. The effect of change in rib stiffness on various modes has been studied.

Free vibration analysis of a square plate with centrally stiffened opening (Fig. 8) analyzed by Paramasivam and Rao by grid framework model and Shastry and Rao [16] by finite element model is carried out and presented in Table 5 in the form of fundamental frequency parameter for simply supported and clamped square plate. The parameter $E_{S} I_{S} / D L$ is taken as 10 , where, $E_{S}$ is the Young's modulus of the stiffener material, $I_{S}$ is the moment of inertia of the stiffener and $D$ is the plate flexural rigidity.

The present results agree well with the result obtained by Shastry and Rao [16]. However the frequencies obtained by Paramasivam and Rao are less compared to the present investigation. This may be due to the fact that Paramasivam and Rao have neglected the torsional rigidity of the stiffeners and the effect of lateral deformation in their analysis. It can be observed that the frequencies obtained by Paramasivam and Rao differ more in the case of unstiffened plate rather than the stiffened plate. The comparison of these results with those in the literature shows the reliability and accuracy of the present formulation and program.

\subsection{Numerical solution}

In the following sections, numerical results for vibration frequencies are studied for simply supported and all edges clamped isotropic stiffened plate having a different number of stiffeners with central square cutout. The stiffened plates are subjected to uni- axial compressive force $N_{X}$ for the first case, and biaxial loading with $N_{X}=N_{y}$ for the second case. The stiffened plate with central square cutout is studied by taking different cutout size ratio $g / a$.

The plate is simply supported at its four edges unless otherwise stated and the data used for its geometry are: $b=600 \mathrm{~mm}, t=1 \mathrm{~mm}, b_{S}=3.31 \mathrm{~mm}$ and $d_{S}=$ $20.25 \mathrm{~mm}$. The other data used are as: $\nu=0.34, E=$ $6.87 \times 10^{4} \mathrm{~N} / \mathrm{mm}, \rho=2.78 \times 10^{-6} \mathrm{Kg} / \mathrm{mm}^{3}$.

As a first case, numerical results for natural frequencies are presented for simply supported and clamped isotropic stiffened square plates having one central stiffener and again three equispaced stiffeners with square central cutouts of different sizes subjected to uniaxial compressive force at the plate boundaries in Figs 9-12. 


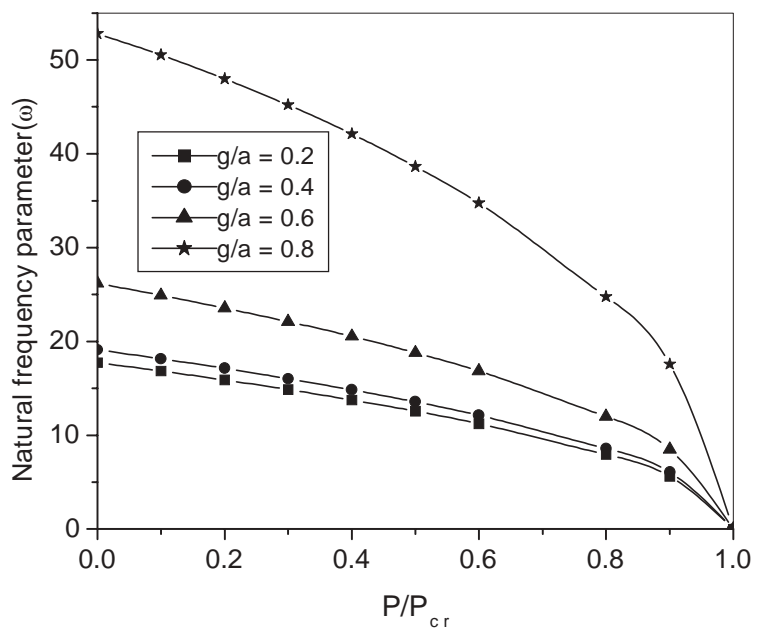

Fig. 9. Frequency parameters for a stiffened square plate having one central stiffener with square cutout subjected to uni-axial compressive forces.

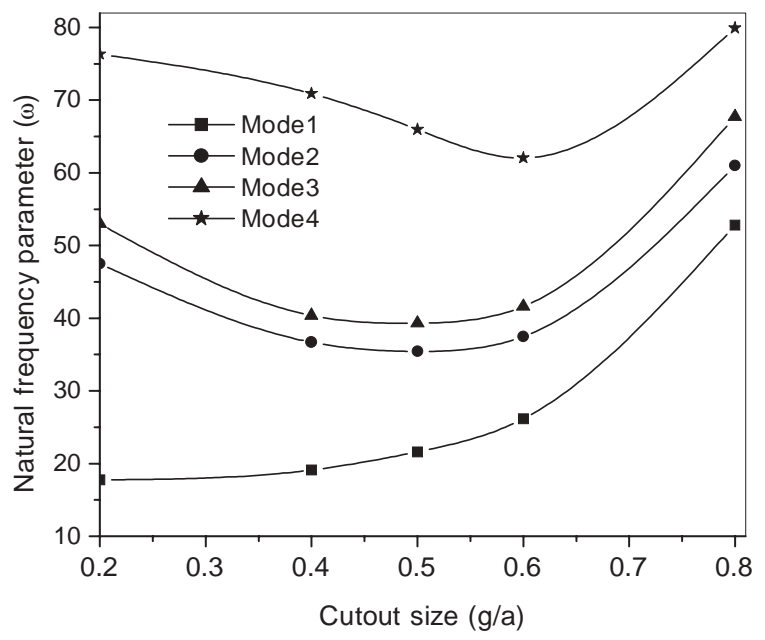

Fig. 10. Frequency parameters for a simply supported stiffened square plate having one central stiffener with square cutouts subjected to uni-axial compressive forces.

It is observed that the behaviour of stiffened plates under in-plane uniform edge loading is the same as that of a complete plate without any cutout. It is also observed from Fig. 9 for one central stiffener and from Fig. 12 for three equispaced stiffeners that the natural frequencies are found to increase with higher number of stiffeners. It is further observed from Fig. 9 that the frequency parameter values increase with the increase of cutout size at different applied load factors $\left(P / P_{c r}\right)$. The rate of increase of frequency from $g / a=0.2$ to 0.4 is less. From $g / a=0.4$ to 0.6 , the rate of increase is more. Again this rate of increase is much more from $g / a=0.6$ to 0.8 . So it can also be concluded

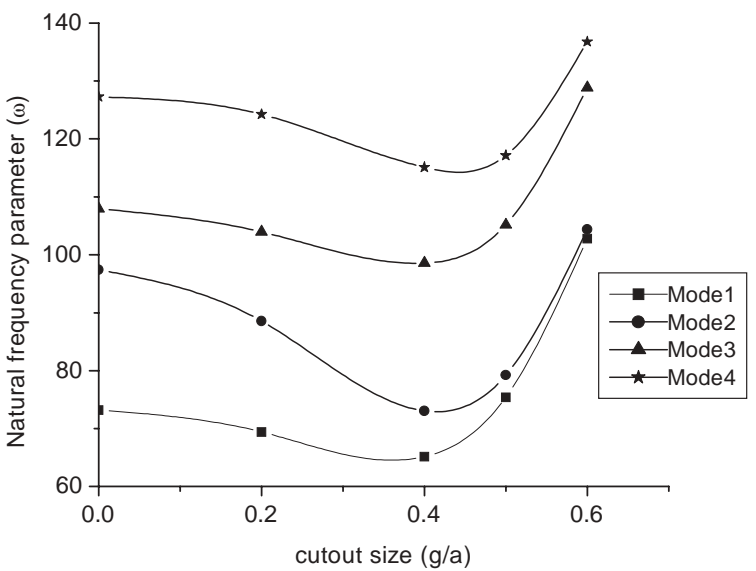

Fig. 11. As Fig. 10, but for clamped edges.

from the above observation that the rate of increase of frequencies also increases with cutout sizes.

The predicted frequency curves for all edges simply supported and clamped stiffened plates at different cutout sizes under uniform in-plane uni-axial load at the plate boundaries in various modes are shown in Figs 10 and 11 respectively. The predicted frequency values will be more in higher modes as expected. The natural frequency variation with cut out size $(g / a)$ for simply supported stiffened square plate having one central stiffener subjected to uni-axial compressive loading is shown in Fig. 10.

The frequency parameter increases for cutout size $(g / a)$ greater than 0.6 for all possible modes. In higher modes these values are observed to be decreased for cutout sizes $(g / a) 0.2$ to 0.6 . For the case of clamped edges, it is observed from the Fig. 11 that frequency parameter increases for cutout size $(g / a)$ greater than 0.4 for all possible modes. Initially the fundamental frequencies of a plate decrease with increasing cutout sizes but subsequently start to increase with increase in the cutout sizes. In higher modes these values are observed to be decreased for cutout sizes $(g / a) 0.2$ to 0.4 .

The effects of bi-axial force on natural frequency for simply supported square stiffened plates for the same dimensions as described above with various cutout sizes are examined here. The results are presented in Figs 13-15. It is observed that the variation of the fundamental frequencies with increased in-plane forces is the same as that of uniaxial force.

The effect of aspect ratios on vibration frequency for stiffened plates with cutouts having one central longitudinal stiffener ( $\delta=0.1$ and $\gamma=10$ ) is studied in this section. In order to study the effect of aspect ratios, 


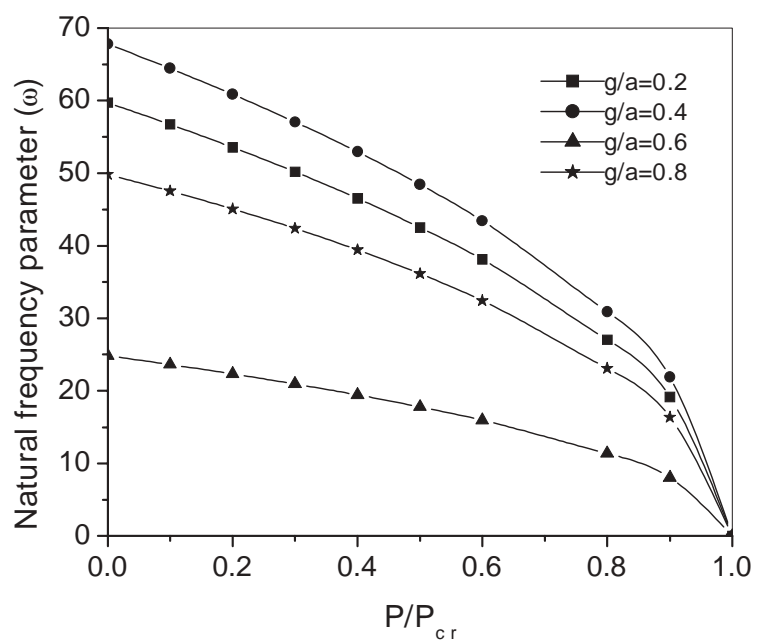

Fig. 12. Frequency parameters for a simply supported stiffened square plate having three equispaced stiffeners with square cutouts subjected to uni-axial compressive forces.

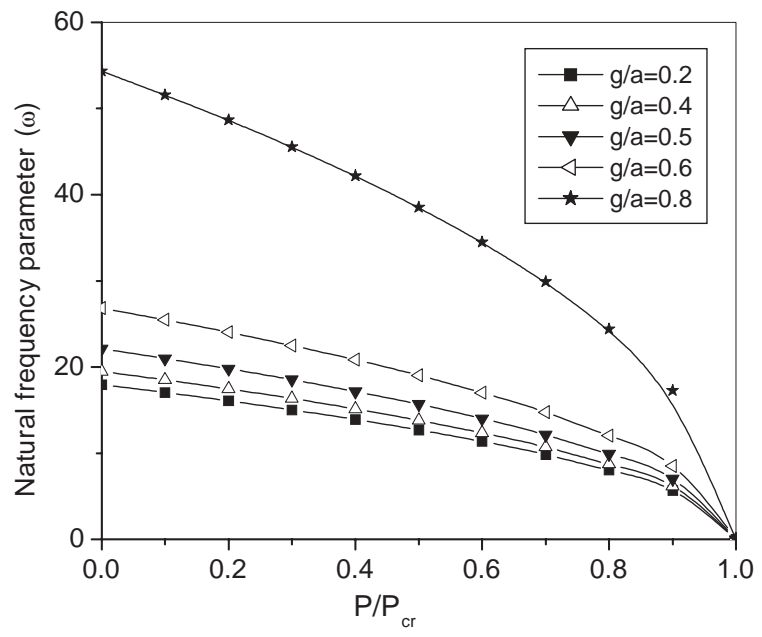

Fig. 13. Frequency parameters for a simply supported stiffened square plate having one central stiffener with square cutouts subjected to bi-axial compressive forces.

the length $(a)$ of the stiffened plate is varied keeping its other parameters unchanged. The study has been done for various aspect ratios of plate $(a / b=1,1.5,2$, $3)$ and aspect ratios of cutout $(g / d=1,1.5,2,3)$ for simply supported and clamped edge conditions. The results obtained are shown in Fig. 16. It can be observed from the figure 16 that vibration frequency parameter decreases with the increase of aspect ratios of plate and cutout -sections both. It can also be observed that the vibration frequency parameter of clamped edge is more than simply supported edge. The effect of aspect ratios of central cutout $(g / d=1,2,3)$ on stiffened

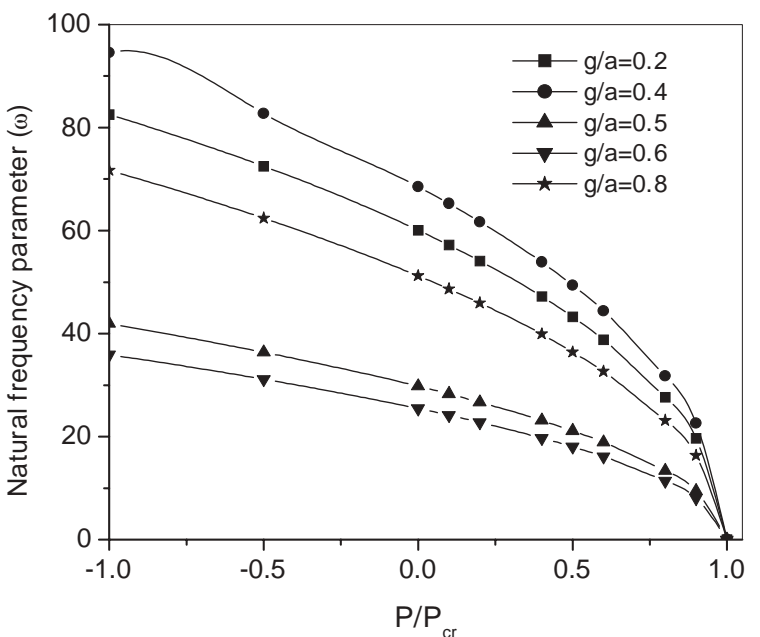

Fig. 14. As Fig. 12, but for bi-axial compressive forces.

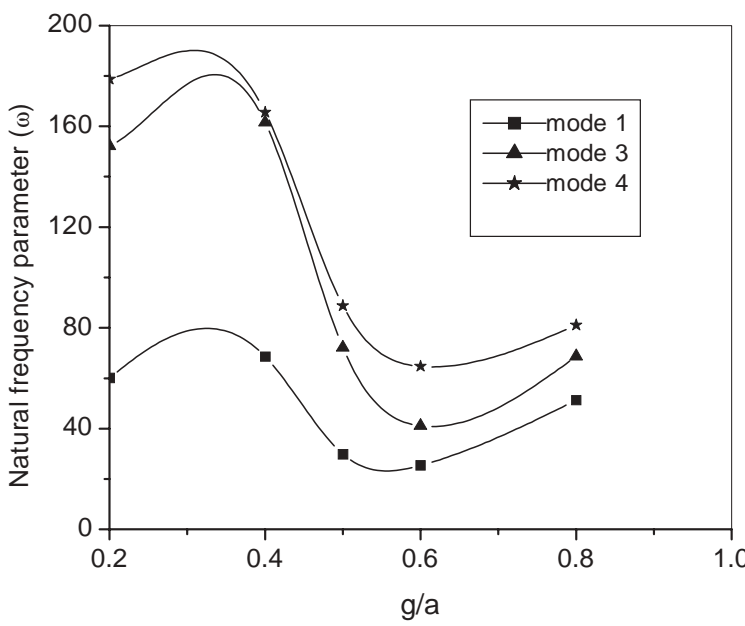

Fig. 15. Frequency parameters for a simply supported stiffened square plates having three equispaced stiffeners with square cutouts subjected to bi-axial compressive forces in various modes.

square plate is studied in Fig. 17 for SSSS and CCCC edge conditions. It is observed from Fig. 17 that vibration frequency decreases as the aspect ratio of cutout increases for the both boundary conditions.

The effect of boundary conditions (SSSS, CCCC, CCSS, SSCC) on the vibration frequencies for stiffened square plates with cutouts $(g / d=2, g / a=0.4, d / b=$ $0.4)$ having one longitudinal central stiffener $(\delta=0.1$ and $\gamma=10$ ) is studied in Fig. 18. It is observed that vibration frequencies increase with the increase of restraint at the edges. Thus the frequency parameter of clamped edges is more than SSSS, CCSS and SSCC boundary conditions. 


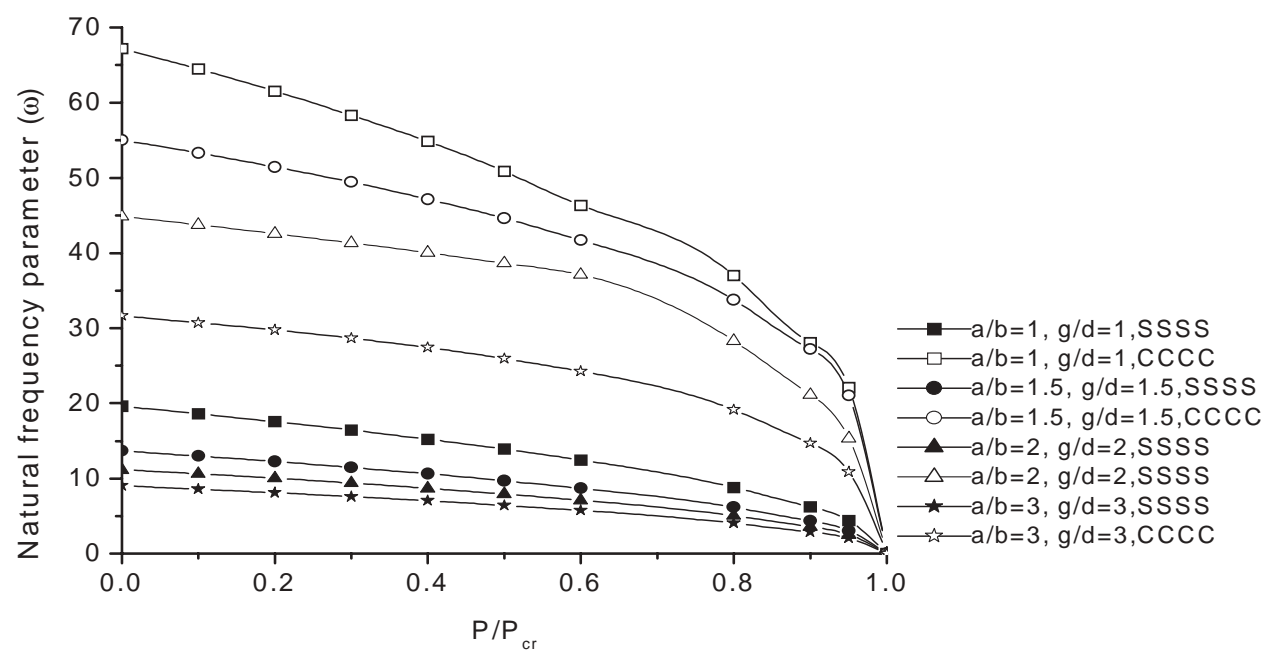

Fig. 16. Effect of aspect ratios on vibration frequency for stiffened plates with cutouts having one central longitudinal stiffener $(\delta=0.1$ and $\gamma=$ 10). $a / b=1,1.5,2,3$ and $g / d=1,1.5,2,3$.

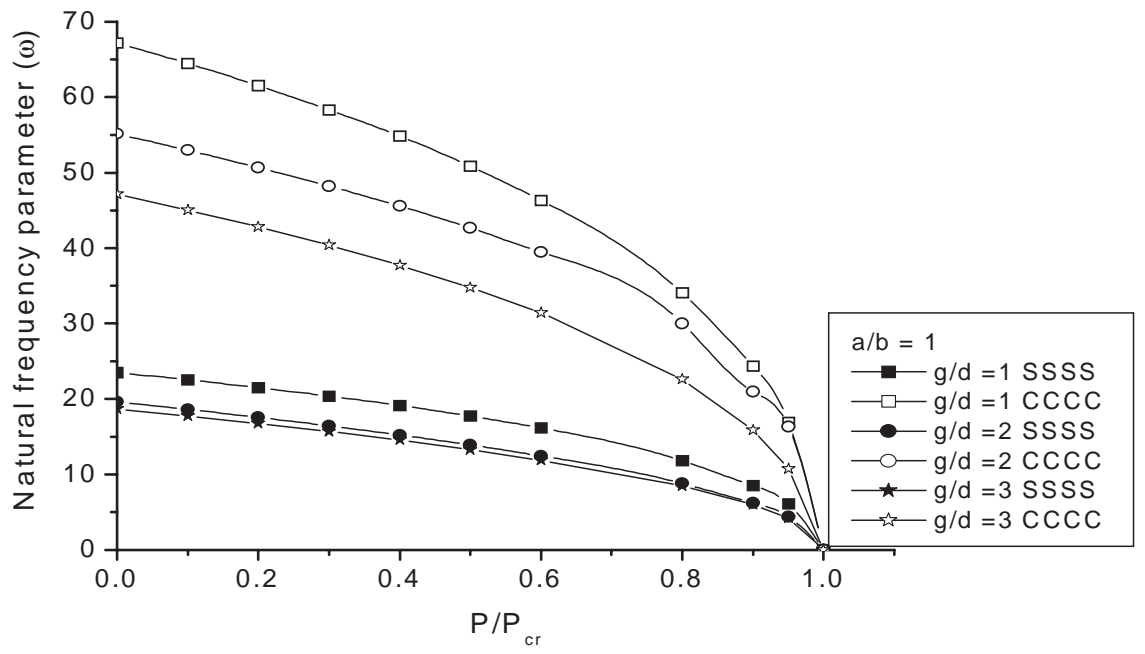

Fig. 17. Effect of aspect ratios of cutout cross-section $(g / d=1,1.5,2,3)$ on vibration frequency for stiffened square plates with cutouts having one central longitudinal stiffener $(\delta=0.1$ and $\gamma=10)$.

\section{Conclusion}

The cutouts have considerable influence on the natural frequencies and mode shapes. The effect is more in higher modes than in the fundamental mode. The natural frequencies obtained increases for higher modes due to increased complexity in the mode shapes.

The present results reflect the initial decreasing trend in frequency with increased cutout size. The effect of shear deformation is more pronounced in the case of clamped plates than in simply supported plates. For CCCC plate, there is a steep increase in frequency with increasing cutout size. The trend of the result for SSSS plate is almost similar to that of CCCC plate, except for its gradient of rise when cutout size increases.

Vibration frequency parameter decreases with the increase of aspect ratios of the plate and the cutout crosssections both. Vibration frequencies increase with the increase of restraint at the edges.

\section{Notations}

$a$

Plate dimension in longitudinal direction

$b \quad$ Plate dimension in the transverse direction 


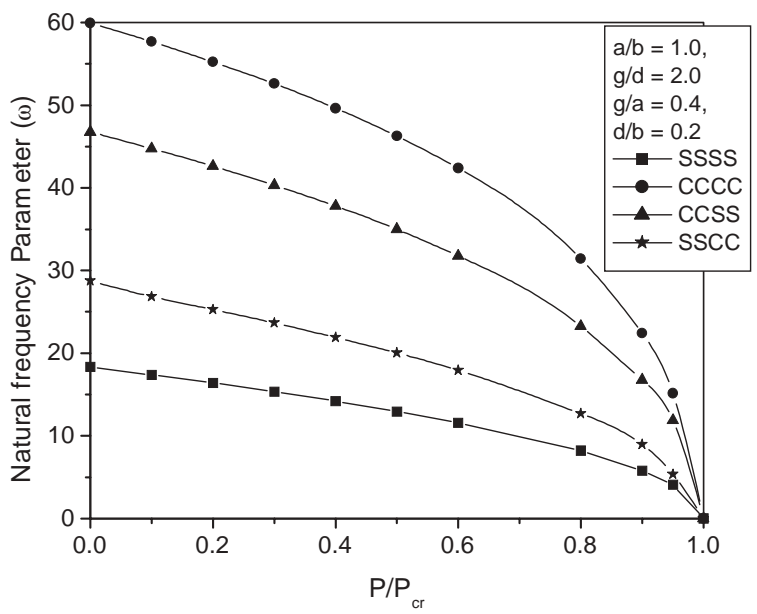

Fig. 18. Effect of boundary conditions on vibration frequency for stiffened square plates with cutouts $(g / d=2, g / a=0.4, d / b=$ $0.4)$ having one central longitudinal stiffener $(\delta=0.1$ and $\gamma=10)$.

\begin{tabular}{|c|c|}
\hline$t$ & Plate thickness \\
\hline$E, G$ & $\begin{array}{l}\text { Young's and shear moduli for the plate } \\
\text { material } \nu \text { Poisson's ratio }\end{array}$ \\
\hline$b_{s}, d_{s}$ & web thickness and depth of a x-stiffener \\
\hline$\xi, \eta$ & Non-dimensional element coordinate \\
\hline$A_{S}$ & Cross sectional area of the stiffener \\
\hline$I_{S}$ & $\begin{array}{l}\text { Moment of inertia of the stiffener cross- } \\
\text { section about reference axis }\end{array}$ \\
\hline$\{q\}_{r}$ & Vector of nodal displacement a rth node \\
\hline$\left[D_{P}\right]$ & Rigidity matrix of plate \\
\hline$\left[D_{S}\right]$ & Rigidity matrix of stiffener \\
\hline$\left[K_{e}\right]$ & Elastic stiffness matrix of plate \\
\hline$\left[K_{S}\right]$ & Elastic stiffness matrix of stiffener \\
\hline$\left[M_{p}\right],\left[M_{S}\right]$ & $\begin{array}{l}\text { Consistent mass matrix of plate, } \\
\text { stiffener }\end{array}$ \\
\hline$\left[K_{G}\right]$ & Geometric stiffness matrix \\
\hline$[N]_{r}$ & Matrix of a shape function of a node $r$ \\
\hline$P_{c r}$ & Critical buckling load \\
\hline$g$ & Cutout length \\
\hline$d$ & cutout width \\
\hline$g / d$ & cutout width ratio \\
\hline$T_{S}$ & Torsional constant \\
\hline$P_{S}$ & $\begin{array}{l}\text { Polar moment of inertia of the stiffener } \\
\text { element }\end{array}$ \\
\hline
\end{tabular}

\section{References}

[1] R. Ali and S.J. Atwal, Prediction of natural frequencies of vibration of rectangular plates with rectangular cutouts, Computer and Structures 12 (1987), 819-823.

[2] C.D. Chang and F.K. Chiang, Vibration analysis of a thick plate with an interior cutout by a finite element method, Journal of sound and Vibration 125(3) (1988), 477-486.

[3] R.B. Corr and A. Jenning, A simultaneous iteration algorithms for symmetric eigenvalue problem, International Journal of Numerical Method Engng 10 (1976), 647-663.

[4] K.Y. Lam and K.C. Hung, Vibration study on plates with stiffened openings using orthogonal polynomials and partitioning method, Computer and Structures 33(3) (1990), 295-301.

[5] P.A.A. Laura, J.C. Utjes and V.H. Palluzzi, On the effect of free, rectangular cutouts along the edge on the transverse vibrations of rectangular plates, Journal of Applied Mechanics 19 (1986), 139-151.

[6] H.P. Lee, S.P. Lim and S.T. Chow, Prediction of natural frequencies of rectangular plates with rectangular cutouts, $\mathrm{Com}$ puter and Structures 36(5) (1990), 861-869.

[7] A.W. Leissa and E.F. Ayoub, Tension buckling of rectangular sheets due to concentrated forces, Journal of Engineering Mechanics. A.S.C.E. 115 (1989), 2749-2762.

[8] I.J. Monahan, P.J. Nemergut and G.E. Maddux, Natural frequencies and mode shapes of plates with interior cutouts, The Shock and Vibration Bulletin 41 (1970), 37-49.

[9] A. Mukherjee and M. Mukhopadhyay, Finite element free vibration of eccentrically stiffened plates, Computer and Structures 30 (1988), 1303-1317.

[10] G. Mundkur, R.B. Bhat and S. Neria, Vibration of plates with cutouts using boundary characteristics orthogonal polynomial functions in the Raleigh-Ritz method, Journal of Sound and Vibration 176(1) (1994), 136-144.

[11] M.D. Olson and C.R. Hazell, Vibration studies of some integral rib stiffened plates, Journal of Sound and Vibration $\mathbf{5 0}$ (1977), 43-61.

[12] P. Paramasivan, Free vibration of square plate with square openings, Journal of Sound and Vibration 30(2) (1973), 173178.

[13] P. Paramshivam and J.K. Sridhar, Free vibration of square plate with stiffened square openings, International Journal of Mechanical Science 15 (1973), 117-122.

[14] J.N. Reddy, Large amplitude flexural vibration of layered composite plates with cutouts, Journal of Sound and Vibration 83(1) (1982), 1-10.

[15] D. Ritchie and Rhodes, Buckling and post-buckling behaviour of plates with holes, Aeronautical Quarterly 24 (1975), 281296.

[16] B.P. Shastry and G.V. Rao, Vibration of thin rectangular plates with arbitrary oriented stiffeners, Computer and Structures 7 (1977), 627-629.

[17] B. Sivasubramonium, G.V. Rao and A. Krishnan, Free vibration of longitudinally stiffened curved panels with cutout, Journal of Sound and Vibration 226(1) (1999), 41-55.

[18] A.K.L. Srivastava, P.K. Datta and A.H. Sheikh, Vibration and dynamic stability of stiffened plates subjected to in-plane harmonic edge loading, International Journal of Structural Stability and Dynamics 2(2) (2002), 185-206.

[19] A.K.L. Srivastava, P.K. Datta and A.H. Sheikh, Buckling and Vibration of stiffened plates subjected to partial edge loading, International Journal of Mechanical Sciences 45(1) (2003), 73-93.

[20] A.K.L. Srivastava, P.K. Datta and A.H. Sheikh, Dynamic stability of stiffened plates subjected to non-uniform harmonic in-plane edge loading, Journal of Sound and Vibration 262(5) (2003), 1171-1189. 

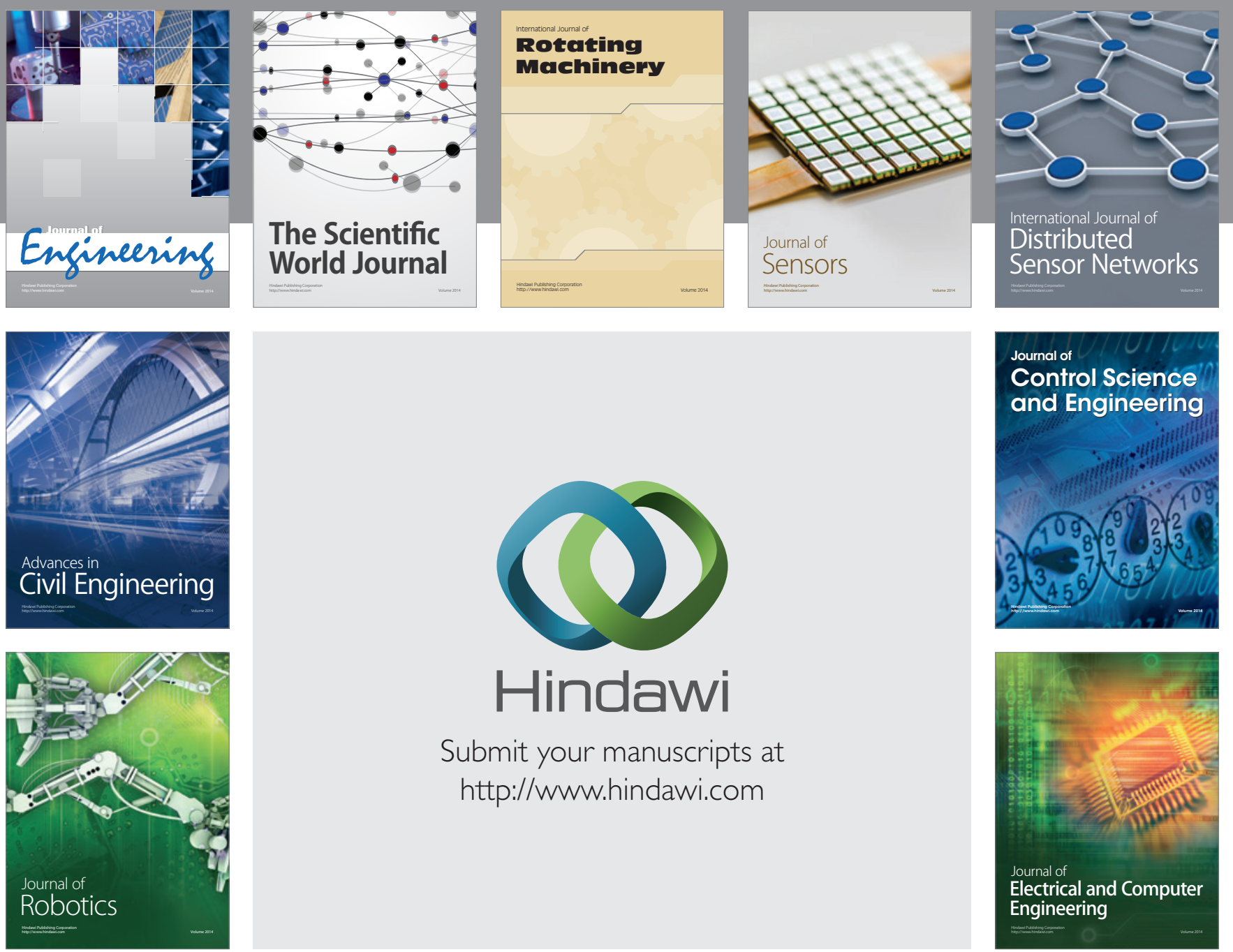

Submit your manuscripts at

http://www.hindawi.com
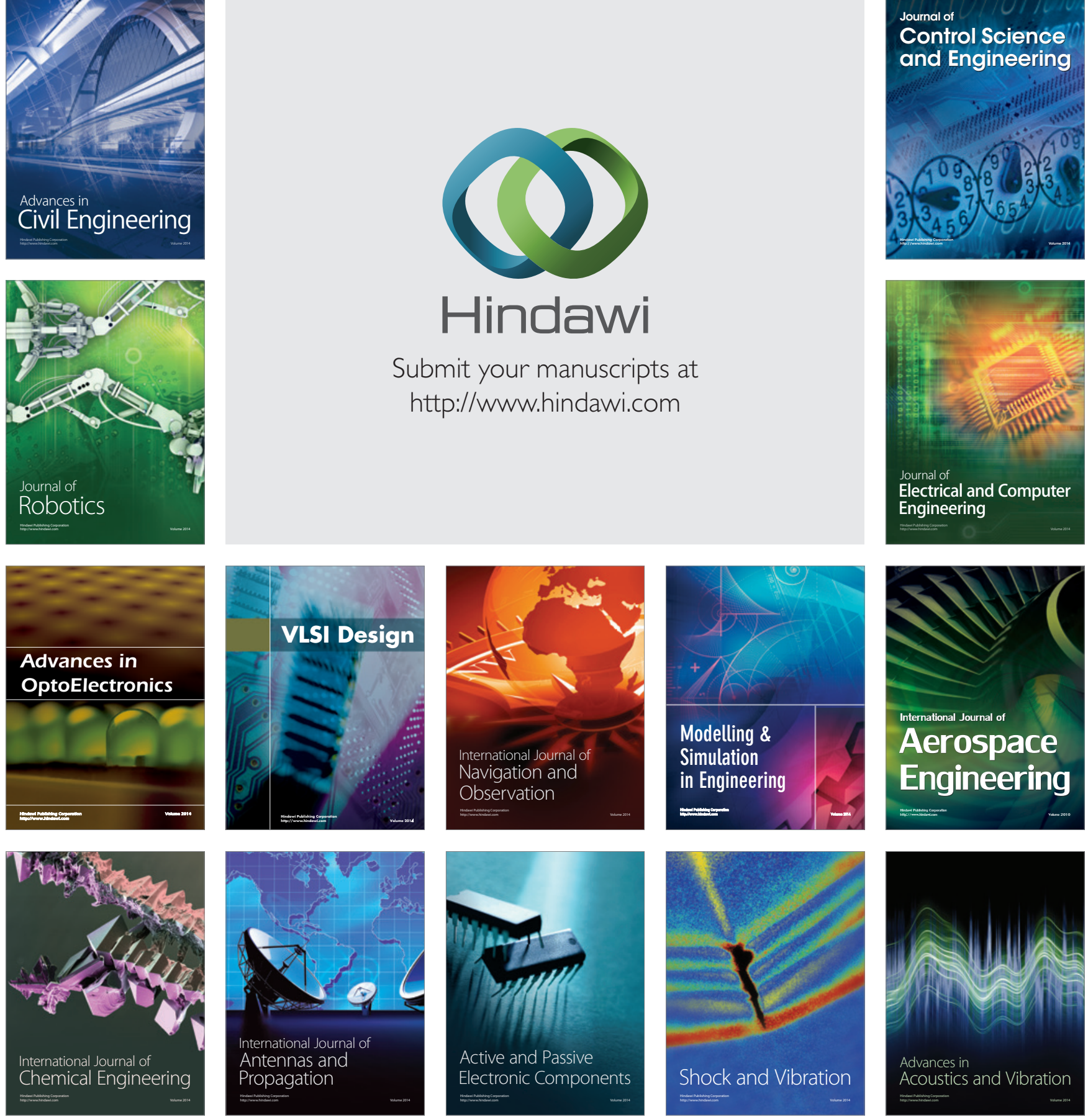